\title{
The importance of flaps in reconstruction of locoregionally advanced lateral skull-base cancer defects: a tertiary otorhinolaryngology referral centre experience
}

\author{
Domen Vozel1 ${ }^{1,2}$, Peter Pukl ${ }^{1}$, Ales Groselj1,2, Aleksandar Anicin ${ }^{1,2}$, Primoz Strojan²,3, \\ Saba Battelino ${ }^{1,2}$ \\ ${ }^{1}$ Department of Otorhinolaryngology and Cervicofacial Surgery, University Medical Centre Ljubljana, Ljubljana, Slovenia \\ ${ }^{2}$ Faculty of Medicine, University of Ljubljana, Ljubljana, Slovenia \\ ${ }^{3}$ Institute of Oncology Ljubljana, Ljubljana, Slovenia
}

Radiol Oncol 2021; 55(3): 323-332.

Received 14 January 2021

Accepted 17 February 2021

Correspondence to: Assoc. Prof. Saba Battelino, M.D., Ph.D., Head of Audiology and Otosurgery, Department of Otorhinolaryngology and Cervicofacial Surgery, University Medical Centre Ljubljana, Zaloška 2, SI-1000 Ljubljana, Slovenia; E-mail: saba.battelino@kclj.si

Disclosure: No potential conflicts of interest were disclosed.

This is an open access article under the CC BY-NC-ND license (http://creativecommons.org/licenses/by-nc-nd/4.0/).

Background. The aim of the study was to identify the value of extensive resection and reconstruction with flaps in the treatment of locoregionally advanced lateral skull-base cancer.

Patients and methods. The retrospective case review of patients with lateral skull-base cancer treated surgically with curative intent between 2011 and 2019 at a tertiary otorhinolaryngology referral centre was made.

Results. Twelve patients with locoregionally advanced cancer were analysed. Lateral temporal bone resection was performed in nine $(75.0 \%)$, partial parotidectomy in six $(50.0 \%)$, total parotidectomy in one $(8.3 \%)$, ipsilateral selective neck dissection in eight (66.7\%) and ipsilateral modified radical neck dissection in one patient (8.3\%). The defect was reconstructed with anterolateral thigh free flap, radial forearm free flap or pectoralis major myocutaneous flap in two patients (17.0\%) each. Mean overall survival was 3.1 years $(S D=2.5)$ and cancer-free survival rate $100 \%$. At the data collection cut-off, $83 \%$ of analysed patients and $100 \%$ of patients with flap reconstruction were alive.

Conclusions. Favourable local control in lateral skull-base cancer, which mainly involves temporal bone is achieved with an extensive locoregional resection followed by free or regional flap reconstruction. Universal cancer registry should be considered in centres treating this rare disease to alleviate analysis and multicentric research.

Key words: temporal bone; microsurgery; parotid region; free tissue flaps; neoplasm staging; ear

\section{Introduction}

Lateral skull-base cancer, which principally involves temporal bone is a rare pathology with an estimated annual incidence of approximately $0.8-6$ per 1 million inhabitants. ${ }^{1,2}$ It presents about $0.2 \%$ of all head and neck cancers. ${ }^{3}$ Metastatic lesions of the lateral skull-base are less frequent than primary tumours and most commonly originate from the breast, pulmonary and renal primaries.
Previous radiotherapy of skull-base (e.g., due to nasopharyngeal cancer), chronic otitis media, human papillomavirus infection, and chlorinated disinfectants are possible risk factors. However, there is a lack of scientific evidence. ${ }^{2,4}$ Lateral skull-base cancer can arise de-novo or result from a malignant transformation of pre-existing benign tumours, such as chondroma to chondrosarcoma. ${ }^{5}$

Despite the advancement of surgical and nonsurgical treatment modalities, the prognosis re- 
mains poor since the reported mean overall survival time does not exceed five years. ${ }^{6}$

\section{Classification of lateral skull-base cancer}

Lateral skull-base cancer can be classified according to the anatomical site into five categories (an adaptation of Homer et al. ${ }^{3}$ ):

- advanced skin cancer of external ear (aEEC); including auricle, concha or periauricular skin,

- advanced parotid cancer (aPC),

- infratemporal fossa and temporomandibular joint cancer,

- primary external auditory canal cancer (EACC) and

- primary middle ear cancer (MEC).

The cancer histological type depends upon the abovementioned site. ${ }^{3}$ Regardless the site, the squamous cell carcinoma is the most common type which accounts for more than $40 \%$ of all primary lateral skull-base cancers, followed by basal cell carcinoma in $10 \%$, adenoid cystic carcinoma in $8-10 \%$ and melanoma is less than $5 \%{ }^{7}$

\section{Clinical presentation of lateral skull-base cancer}

The clinical presentation of lateral skull-base cancer is not pathognomonic since it can mimic the chronic inflammatory diseases, such as chronic otitis media, chronic otitis externa, necrotising otitis externa or vasculitis (e.g., granulomatosis with polyangiitis). For that reason, bloody otorrhoea, hearing loss, bleeding, chronic otalgia, facial swelling or palsy and mass should be attributed to the lateral skullbase cancer until proven otherwise. Granulation tissue, nonhealing ulcer or erosion should be elucidated by histopathological examination. In case of progressive pain in the temporomandibular joint area, trismus, facial pain and fullness or subtle mass immediately above zygoma, suspicion of infratemporal fossa cancer should be raised. ${ }^{3}$

\section{Diagnosis of lateral skull-base cancer}

Diagnosis of lateral skull-base cancer mainly comprises histopathological or cytopathological verification, high-resolution computed tomography (CT) and magnetic resonance imaging (MRI) of skull-base and adjacent structures (i.e. parotid region, neck), neck CT or ultrasonography (US) and further evaluation of signs of systemic cancer spread (e.g., abdominal CT/US, chest CT/x-ray, positron emission tomography-CT). ${ }^{3}$
TABLE 1. Modified Pittsburgh staging system 8,9

\begin{tabular}{|c|c|}
\hline \multicolumn{2}{|c|}{ T assessment } \\
\hline $\mathrm{Tl}$ & $\begin{array}{l}\text { Tumour limited to external auditory canal without } \\
\text { bony erosion or evidence of soft tissue involvement }\end{array}$ \\
\hline T2 & $\begin{array}{l}\text { Tumour with limited external auditory canal bone } \\
\text { erosion (not full thickness) or limited }(<0.5 \mathrm{~cm}) \text { soft- } \\
\text { tissue involvement }\end{array}$ \\
\hline Т3 & $\begin{array}{l}\text { Tumour eroding osseous external auditory canal } \\
\text { (full thickness) with limited }(<0.5 \mathrm{~cm} \text { ) soft tissue } \\
\text { involvement or tumour involving the middle ear } \\
\text { and/or mastoid }\end{array}$ \\
\hline $\mathrm{T} 4$ & $\begin{array}{l}\text { Tumour eroding cochlea, petrous apex, medial wall } \\
\text { of the middle ear, carotid canal, jugular foramen or } \\
\text { dura, or with extensive soft tissue involvement }(>0.5 \\
\mathrm{cm} \text { ) such as involvement of temporomandibular } \\
\text { joint or styloid process, or evidence of facial paresis }\end{array}$ \\
\hline \multicolumn{2}{|c|}{$\mathrm{N}$ assessment } \\
\hline NO & No regional lymph node metastasis \\
\hline $\mathrm{NI}$ & Regional lymph node metastasis \\
\hline \multicolumn{2}{|c|}{$M$ assessment } \\
\hline MO & No distant metastasis \\
\hline Ml & Distant metastasis \\
\hline \multicolumn{2}{|c|}{ Stage group } \\
\hline 1 & TINOMO* \\
\hline$\|$ & T2NOMO* \\
\hline III & T3NOMO‡, T1N1MO‡ \\
\hline IV & T4NOMO‡, T2-4NIMO‡, T1-4NO-1MI ${ }^{\dagger}$ \\
\hline
\end{tabular}

The TNM assessment is based on the clinical examination and imaging findings. This staging system has been applied to assess primary external auditory canal cancer and primary middle ear cancer.' Middle ear cancer is assessed as at least T3, therefore always locoregionally advanced.

$T=$ tumour; $N=$ regional lymph node metastasis; $M=$ distant metastasis: " = localised cancer; ${ }^{\ddagger}=$ locoregionally advanced cancer; ${ }^{\dagger}=$ systemically advanced cance

The clinical examination and diagnostic procedures enable the assessment of tumour stage according to the TNM classification system. Primary tumour $(\mathrm{T})$, regional lymph node metastases $(\mathrm{N})$ and distant metastases (M) can be assessed according to the primary tumour site: e.g. for skin carcinoma of the head and neck, major salivary glands, malignant melanoma of the skin, tumours of bone and soft tissue. EACC and MEC deserve particular emphasis in T, N and M assessment as the AJCC and UICC grading systems do not include these cancer types. EACC should be graded with the modified Pittsburgh staging system (Table 1) ) $^{8,9}$, which has the highest prognostic accuracy. ${ }^{10}$ This staging system should be used in pathological TNM staging also. ${ }^{11}$ MEC has been graded with many staging systems including modified Pittsburgh staging system ${ }^{18,9}$ and Stell and McCormick grading system. ${ }^{12}$ 


\section{Treatment of lateral skull-base cancer}

After the diagnosis has been established, the patient should be presented at the multidisciplinary tumour board to determine the treatment modalities and goals. ${ }^{3}$ In our tertiary referral centre, the board, usually consists of an otorhinolaryngologist subspecialised in otologic and lateral skull-base surgery, otorhinolaryngologist subspecialised in head and neck surgery and free flap reconstruction, radiation oncologist and medical oncologist.

The best prognosis of lateral skull-base cancer is achieved with the radical surgical treatment, which depends on the tumour's extent and presence of regional lymph node metastasis. ${ }^{7}$ Therefore, it can include for an example wide local excision of the tumour, temporal bone resection (lateral, subtotal or total), parotidectomy (superficial or total), neck dissection (selective, modified radical, radical) and temporomandibular joint resection. When the histopathological examination of the resected specimen implies an increased risk of local/regional tumour re-appearance, adjuvant treatment must be considered. Other non-surgical treatment modalities (i.e., radiotherapy, systemic therapy), when indicated, can significantly improve the rate of local and/or regional control. ${ }^{7}$

Since the extensive surgery of locoregionally advanced lateral skull-base cancer results in large tissue defects, the reconstruction should be planned immediately. An assortment of free flaps can be considered to aid the reconstruction such as radial forearm free flap (RFFF), deep inferior epigastric perforator flap, latissimus dorsi free flap and anterolateral thigh free flap (ALT). ${ }^{3}$ ALT is considered a workhorse in lateral skull-base reconstruction since it provides an adequate tissue bulk to fill the tissue defect. Additionally, the donor site (i.e., thigh wound) can be used to harvest fascia lata to reconstruct dural defect or statically suspend the oral commissure if the facial nerve has been sacrificed. Moreover, ALT can be harvested as chimaeric, i.e. incorporating the lateral cutaneous femoral nerve, which can be used as interposition nerve graft in facial nerve reanimation. It is recommended that facial nerve reanimation should be initiated at primary surgery. However, in certain circumstances (i.e., peripheral arterial occlusive disease, head and neck scarring after the previous radiotherapy) free flaps cannot be used or have failed to reconstruct the defect. ${ }^{3}$ For that reason, regional flaps such as the pectoralis major myocutaneous flap (PM) should be harvested. $3,13,14$
The treatment of lateral-skull base cancer should be reserved for highly specialised centres. This manuscript aims to provide an experience of a single tertiary otorhinolaryngology referral centre in the treatment of this pathology, emphasising the role of regional and free flaps in reconstruction after resection of locoregionally advanced lateral skull-base cancer.

\section{Patients and methods}

The study protocol was approved by the Institutional Committee for Medical Ethics and the Protocol Review Board (ERIDNPVO-0012/2020, 29.7.2020). The study was performed according to the ethical standards of the responsible institutional review board on human experimentation and with the Helsinki Declaration. Patients provided written, informed consent at the admission.

\section{Patients' data acquisition}

A retrospective case review of patients treated at the Institute of Oncology Ljubljana, Slovenia and/or Department of Otorhinolaryngology and Cervicofacial Surgery, University Medical Centre, Ljubljana, Slovenia was performed. Inclusion criteria were:

- time of cancer diagnosis between the January $1^{\text {st }} 2011$ and December $31^{\text {st }} 2019$,

- international classification of diseases, $10^{\text {th }}$ revision (ICD-10) diagnoses:

- C07 (lat. neoplasma malignum glandulae parotideae),

- C30.1 (lat. neoplasma malignum auris mediae),

- C41.0 (lat. neoplasma malignum ossium cranii et faciei),

- C43.2 (lat. melanoma malignum auris et meatus acustici externi),

- C44.2 (lat. neoplasma malignum cutis auris et meatus acustici externi) or

- C49.0 (lat. neoplasma malignum textus connexivi et mollis capitis, faciei et colli).

- surgical treatment with curative intent.

The data were collected from the Cancer Registry, Slovenia, databases of Department of Otorhinolaryngology and Cervicofacial Surgery, University Medical Centre Ljubljana, Slovenia and Institute of Oncology Ljubljana, Slovenia. 
Data of 177 patients collected from Cancer Registry of Republic of Slovenia with:

- ICD-10 diagnoses: C07, C30.1, C41.0, C43.2, C44.2, C49.0 and

- any histopathological diagnosis and

- treated at Institute of Oncology Ljubljana and/or Department of

Otorhinolaryngology and Cervicofacial Surgery and

- treated between 2011-2019 and

- any therapeutic modality.

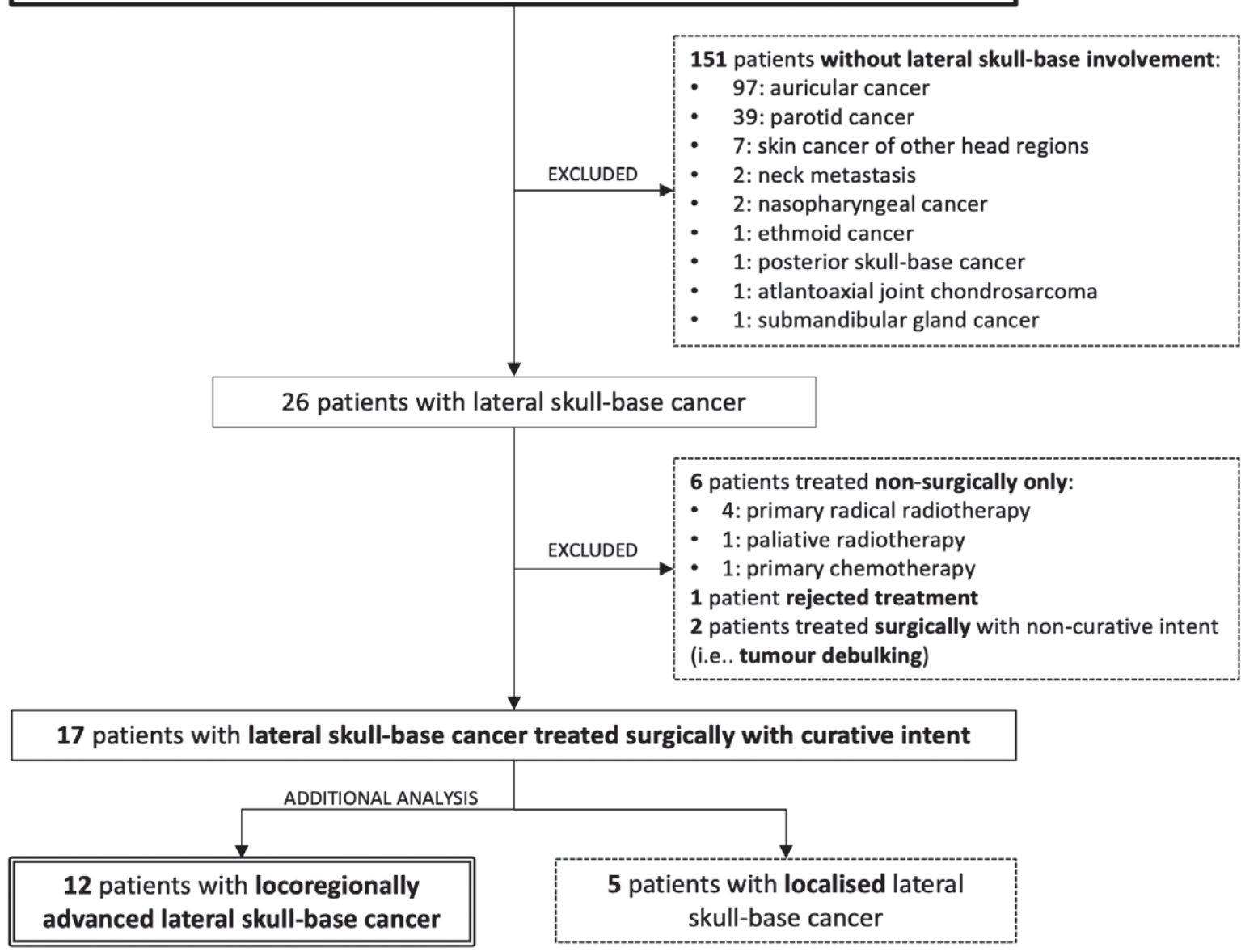

$I C D=$ International statistical classification of diseases and related Health problems $10^{\text {th }}$ revision

FIGURE 1. Data acquisition flowchart of patients with lateral skull-base cancer. Data of 177 patients were thoroughly analysed using Cancer Registry of the Republic of Slovenia and databases of Department of Otorhinolaryngology and Cervicofacial Surgery, University Medical Centre Ljubljana, Slovenia and Institute of Oncology Ljubljana, Slovenia. The majority of excluded patients suffered from auricular or parotid cancer without lateral skull-base involvement. Additional analysis was performed on the data of locoregionally advanced cancer.

\section{Patients' data analysis}

Included patients were analysed for the gender, age, symptoms at presentation, date of cancer diagnosis, ICD-10 diagnosis, histology, clinical and pathological TNM stage (utilising the University of Pittsburgh TNM staging system (Table 1) for EACC and MEC or 8th edition of UICC staging system for aEEC and aPC), tumour localisation according to the abovementioned classification, preoperative head and neck imaging modalities and preoperative pure tone audiometry. Pure tone average was calculated for the affected ear (for bone and air conduction) as an average of hearing levels of pure tone audiometry at speech frequencies (i.e., $500 \mathrm{~Hz}$, $1000 \mathrm{~Hz}, 1500 \mathrm{~Hz}, 2000 \mathrm{~Hz}, 3000 \mathrm{~Hz}$ and $4000 \mathrm{~Hz}$ ). The average air-bone gap was calculated from pure tone average for bone and air conduction.

Moreover, date of surgery, treatment modalities employed (i.e., surgery, radiotherapy, systemic 
therapy), residual tumour classification according to the UICC staging system (i.e., R0 no residual tumour, R1 microscopic residual tumour and R2 macroscopic residual tumour), and date of the last recorded check-up or date and reason of death were analysed. Cancer recurrence was defined as the re-appearance of cancer in the surgical bed after the treatment was completed, and cancer was considered cured.

Listed data was used to analyse age at the cancer diagnosis, age at death, postoperative follow-up period and survival. Local control and overall survival crude rates and estimates using the KaplanMeier method were determined. Patients were considered cancer-free if there was no cancer recorded at five years after the surgery.

Statistical analysis was performed using Microsoft Excel for Mac (version 16 and later, Microsoft Corporation, Redmond, Washington) and SPSS (version 23, IBM Corp., Armonk, New York). Basic descriptive statistics were reported with means $(M)$ and standard deviations (SD) and a $p$-value $(p)$ below 0.05 was considered statistically significant.

\section{Results}

\section{Study flowchart, demographics and clinical presentation}

Data collection was cut off on September $14^{\text {th }} 2020$. Initially, 177 patients were included in the study. Seventeen $(10 \%)$ patients with lateral skull-base cancer were treated with curative intent between 2011 and 2019, and in 12 of them, the tumour was locally advanced (Table 2).

At the admission, $12(71 \%)$ patients reported discharge, $10(59 \%)$ crusting or nonhealing lesion, $10(59 \%)$ pain, $6(35 \%)$ bleeding, $5(29 \%)$ hearing loss and $2(12 \%)$ itching. A patient $(6 \%)$ with aPC extending to the lateral skull-base reported the unilateral facial muscle weakness. None reported vertigo or other symptoms related to other cranial nerves involvement.

\section{Tumour characteristics and localisation}

There was no left to right predominance (53\% left and $47 \%$ right). Seven patients ( $41 \%$ ) had an aEEC. Basal cell carcinoma was present in four (57\%) and squamous cell carcinoma in three $(43 \%)$ patients (Table 2).

Six patients (35\%) suffered from EACC. The latter was classified as C44.2 in $100 \%$. The cancer was

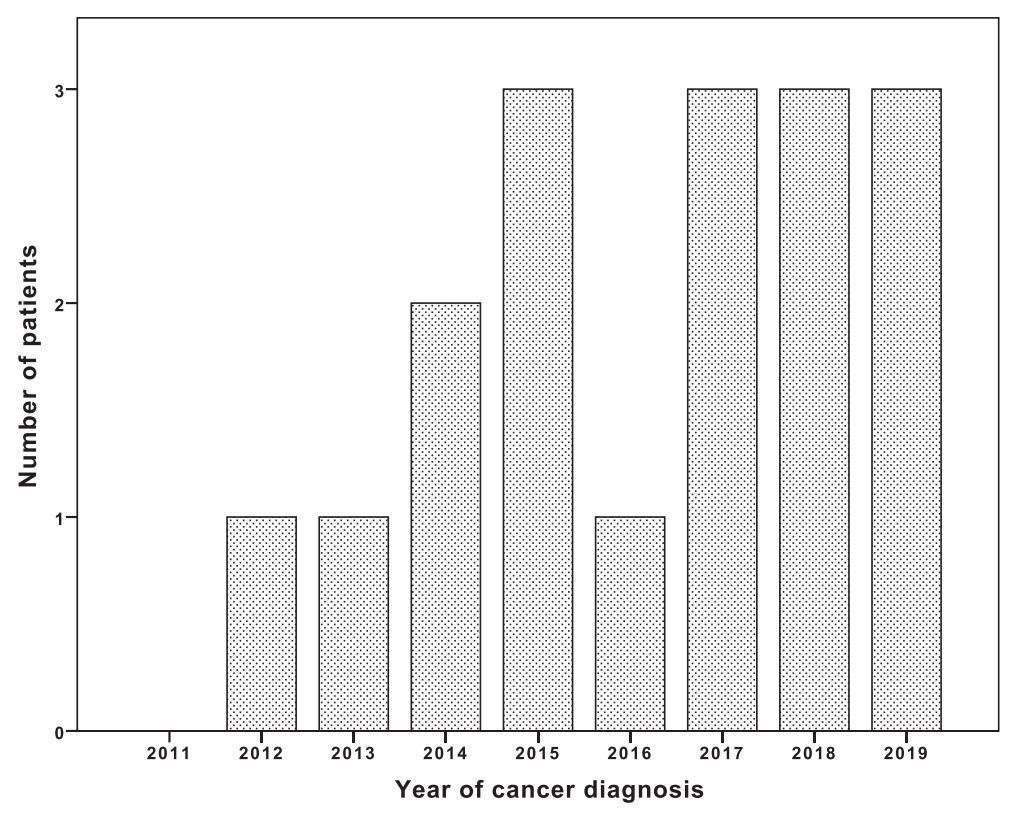

FIGURE 2. Barchart of seventeen patients with lateral skull-base cancer treated with curative intent between 2011 and 2019.

squamous cell carcinoma in three $(50 \%)$, basal cell carcinoma in two $(33 \%)$ and adenoid cystic carcinoma in one patient $(17 \%)$.

Both $(12 \%)$ MECs were squamous cell carcinoma and two $(12 \%)$ aPCs were mucoepidermoid carcinoma (50\%) and adenocarcinoma (50\%).

Disregarding the tumour localisation squamous cell carcinoma was the most common type (8 patients, $47 \%$ ), followed by basal cell carcinoma (6 patients, 35\%), and others (adenoid cystic carcinoma $(6 \%)$, mucoepidermoid carcinoma $(6 \%)$ and adenocarcinoma (6\%).

\section{Locoregionally advanced lateral skull- base cancer}

In twelve patients $(71 \%)$, the tumour was locoregionally advanced (i.e., grades III and IV). Preoperative skull-base imaging (i.e., CT or MRI) was performed in all of them. Pure tone average for air and bone conduction and air-bone gap could be calculated in eight patients (67\%). Average value of pure tone average was $69 \mathrm{~dB}(S D=39 \mathrm{~dB})$ for air conduction, $43 \mathrm{~dB}(S D=19 \mathrm{~dB})$ for bone conduction and average air-bone gap was $26 \mathrm{~dB}(S D=20 \mathrm{~dB})$.

The primary surgery was the only treatment modality in five patients $(42 \%)$ patients. Other treatment modalities employed were salvage surgery in three $(25 \%)$ patients (i.e., one after radiotherapy, one after radiotherapy and electrochemotherapy, 
TABLE 2. Dataset of patients with lateral skull-base cancer treated between 2011 and 2019

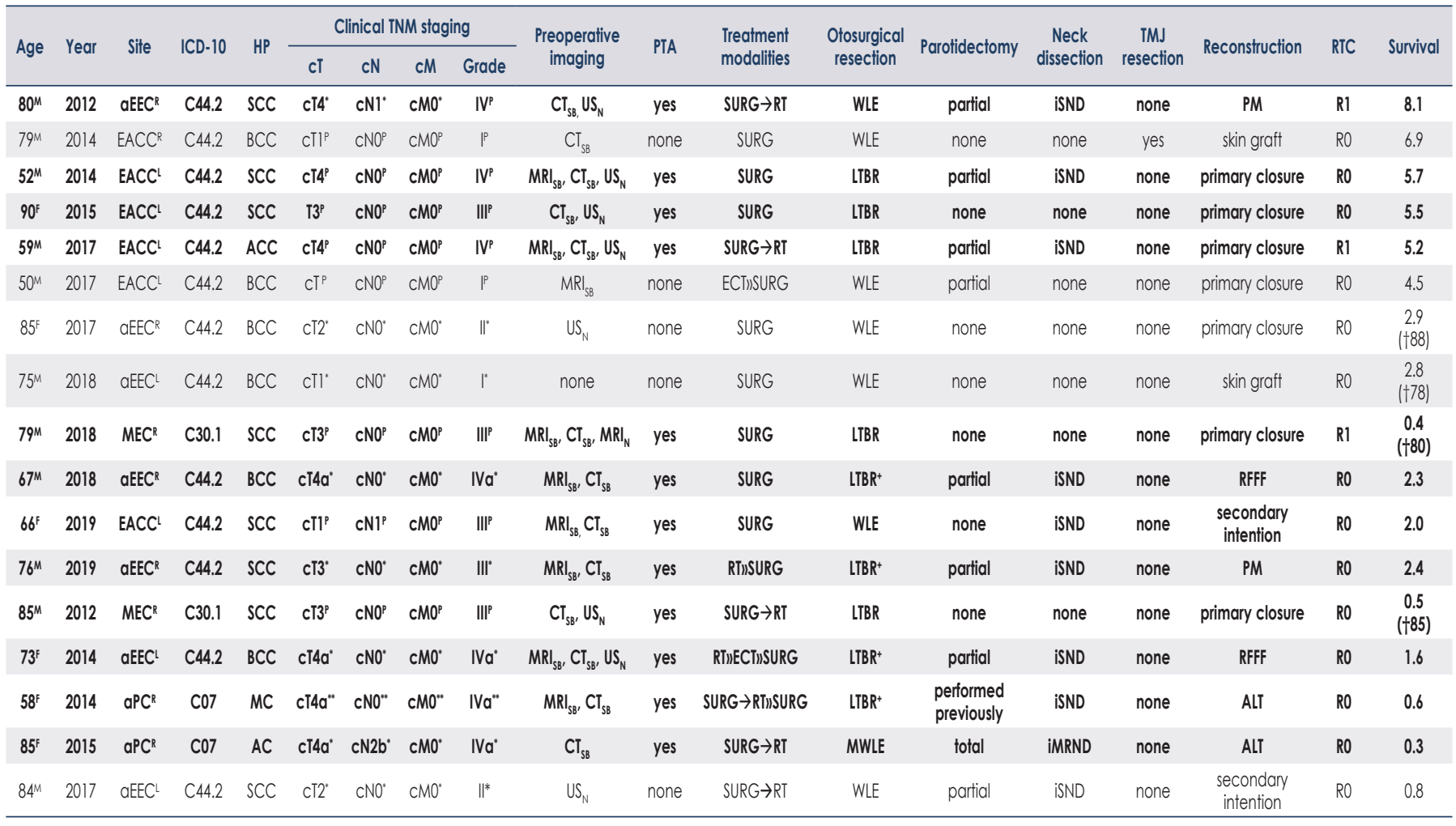

Locoregionally advanced cancer is shown in bold. Age and survival are depicted in years

$\mathrm{AC}=$ adenocarcinoma; $\mathrm{ACC}=$ adenoid cystic carcinoma; $\mathrm{aEEC}=$ advanced skin cancer of external ear (including auricle, concha or periauricular skin); $\mathrm{Age}=$ age at the time of cancer diagnosis; $\mathrm{ALT}=$ anterolateral thigh free flap; $\mathrm{aPC}=$ advanced parotid cancer; $\mathrm{BCC}=$ basal cell carcinoma; $\mathrm{CT}_{\mathrm{SB}}=$ skull-base computed tomography; $E A C C=$ primary external auditory canal cancer; $E C T$ = electrochemotherapy; $E C T$ IISURG = ECT was performed with primary curative intent and surgery as salvage; $F=$ female; $\mathrm{HP}=$ histopathological diagnosis; ICD-10 = international classification of diseases, $10^{\text {th }}$ revision; iMRND = ipsilateral modified radical neck dissection; iSND = ipsilateral selective neck dissection; $L=$ left; $L T B R=$ lateral temporal bone resection; $\mathrm{LTBR}^{+}=$lateral temporal bone resection with wide local excision; $M=$ male; $M$ WLE $=$ mastoidectomy with wide local

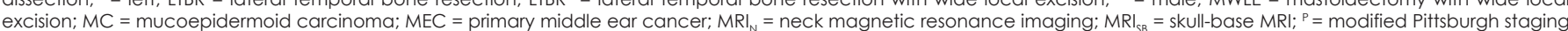

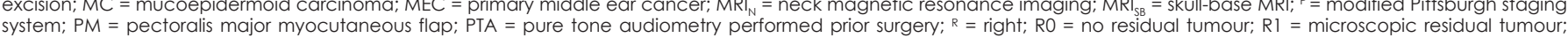
RFFF = radial forearm free flap; RT = radiotherapy; RTC = residual tumour classification according to the UICC staging system; SCC = squamous cell carcinoma;

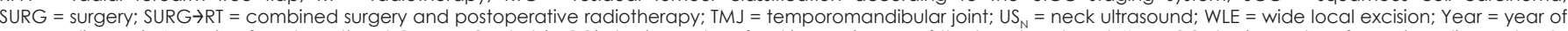
cancer diagnosis; " = Union for International Cancer Control (UICC) staging system for skin carcinoma of the head and neck " = UICC staging system for major salivary glands; t with a number $=$ an age at death

one after surgery and radiotherapy) and postoperative radiotherapy in four patients (33\%) (Table 2). None received postoperative chemotherapy.

Wide local excision only was performed in two $(16.7 \%)$, mastoidectomy in one $(8.3 \%)$ and lateral temporal bone resection with obliteration in nine patients (75\%). Additional partial parotidectomy was performed in six (50\%), total parotidectomy in one $(8.3 \%)$, ipsilateral selective neck dissection of regions II-IV in eight $(66.7 \%)$ and ipsilateral modified radical neck dissection including resection of the sternocleidomastoid muscle in one patient $(8.3 \%)$.

The post-resection lateral skull-base defect was reconstructed with primary closure in five (41.7\%) and flap in six (50\%) patients with locoregionally advanced cancer. The wound was left to heal by secondary intention in one $(8.3 \%)$ patient with T1N1M0 (stage III) EACC.

Reconstruction with ALT (Figure 3), RFFF (Figure 4) and PM (Figure 5) was performed in two patients each. There was no flap failure. In five patients $(83 \%)$ with flap reconstruction, the resection was R0. Postoperative photon radiotherapy with a dose of 60 Gy and 64 Gy in 2 Gy daily fractions was performed in two patients, including the one with R1 resection.

\section{Local control and survival after locoregionally advanced lateral skull- base cancer resection}

The mean follow-up time (i.e., the mean overall survival time) of twelve patients after locoregion- 


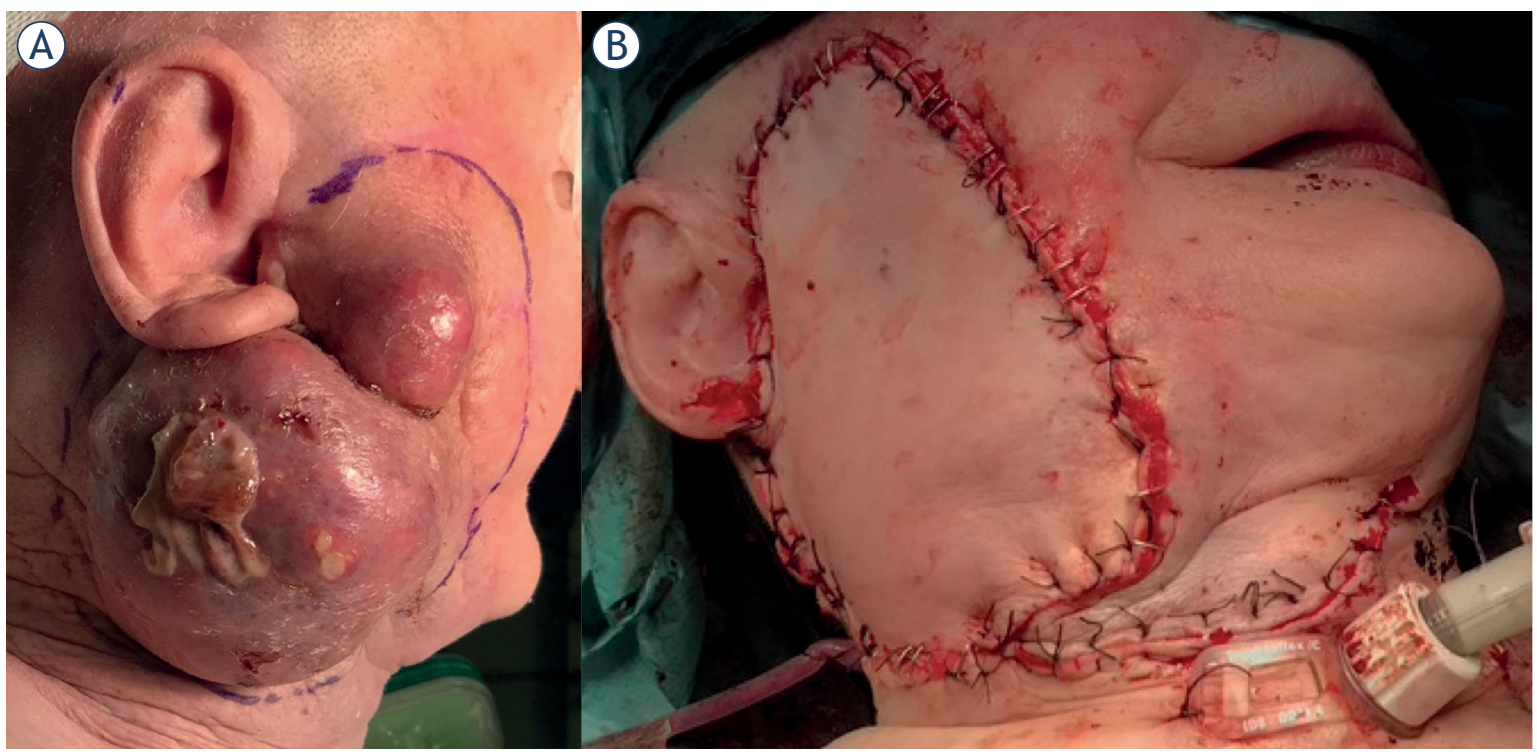

FIGURE 3. 85-year old female with locoregionally advanced parotid adenocarcinoma (i.e., parotid metastasis after incomplete temporal skin adenocarcinoma cancer resection) extending to the right external auditory canal and lateral skull-base. The resection margin is outlined (A). Primary surgery involving mastoidectomy with wide local excision, total parotidectomy, modified radical neck dissection, temporary tracheostomy, static suspension of oral commissure with fascia lata and anterolateral thigh free flap reconstruction were performed (B).

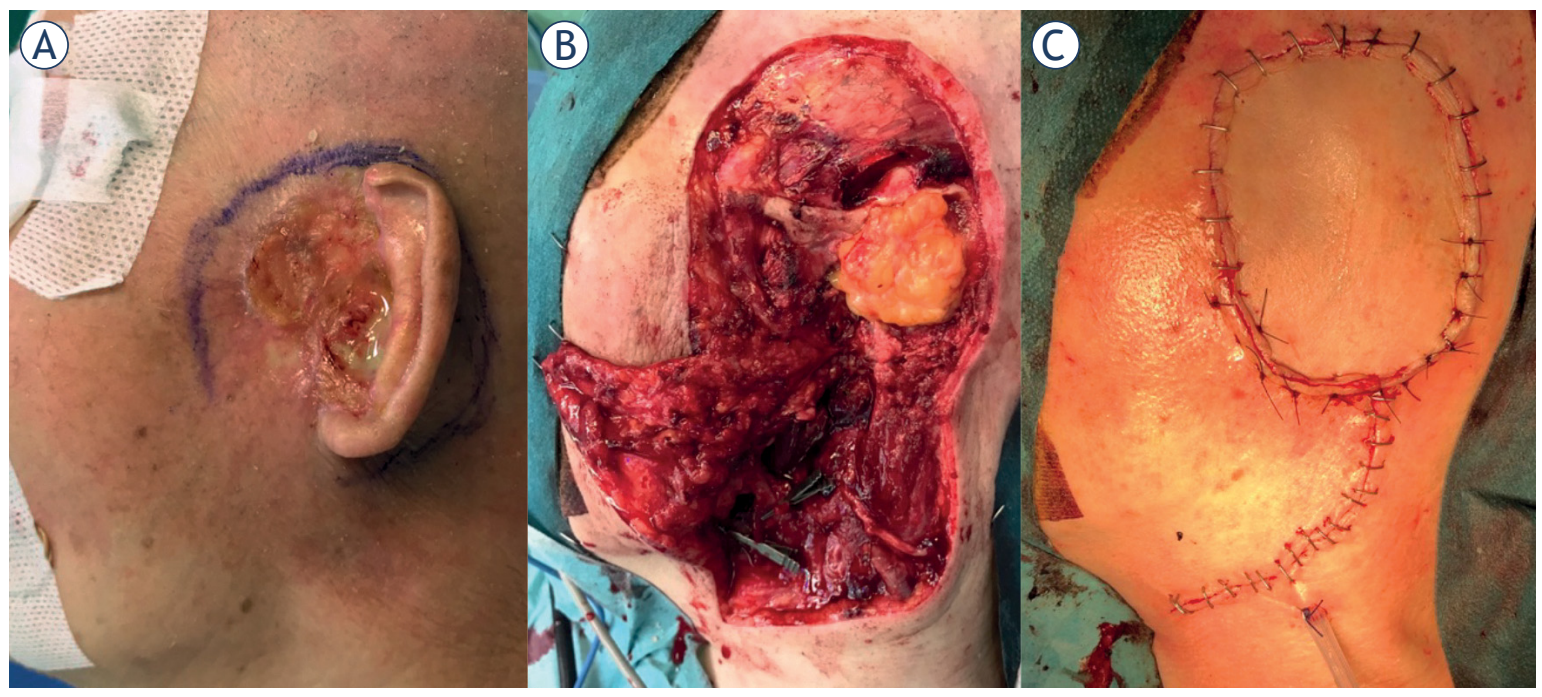

FIGURE 4. 73-year old female with locoregionally advanced external ear basal cell carcinoma extending to the left lateral skullbase. The resection margin is outlined (A). Salvage surgery (i.e., after primary radical radiotherapy and electrochemotherapy) involving lateral temporal bone resection with fat obliteration, with wide local excision, partial parotidectomy, ipsilateral selective neck dissection (B) and radial forearm free flap reconstruction (C) were performed.

ally advanced lateral skull-base cancer resection was 2.9 years $(S D=2.6$ years, range: 0.3 years -8.1 years). At the data collection cut-off date, ten of these patients $(83 \%)$ were alive and had no cancer recurrence (Table 2) (Figure 6A). Two patients $(27 \%)$ died, but no recurrence was detected. One patient died with MEC since the resection was R1, and the patient did not receive planned postoperative radiotherapy due to generalised weakness. One patient after R0 resection of MEC died due to comorbidities. The survival was $100 \%$ in patients treated with flap reconstruction and $67 \%$ in patients treated with other reconstruction modalities (Figure 6B). 


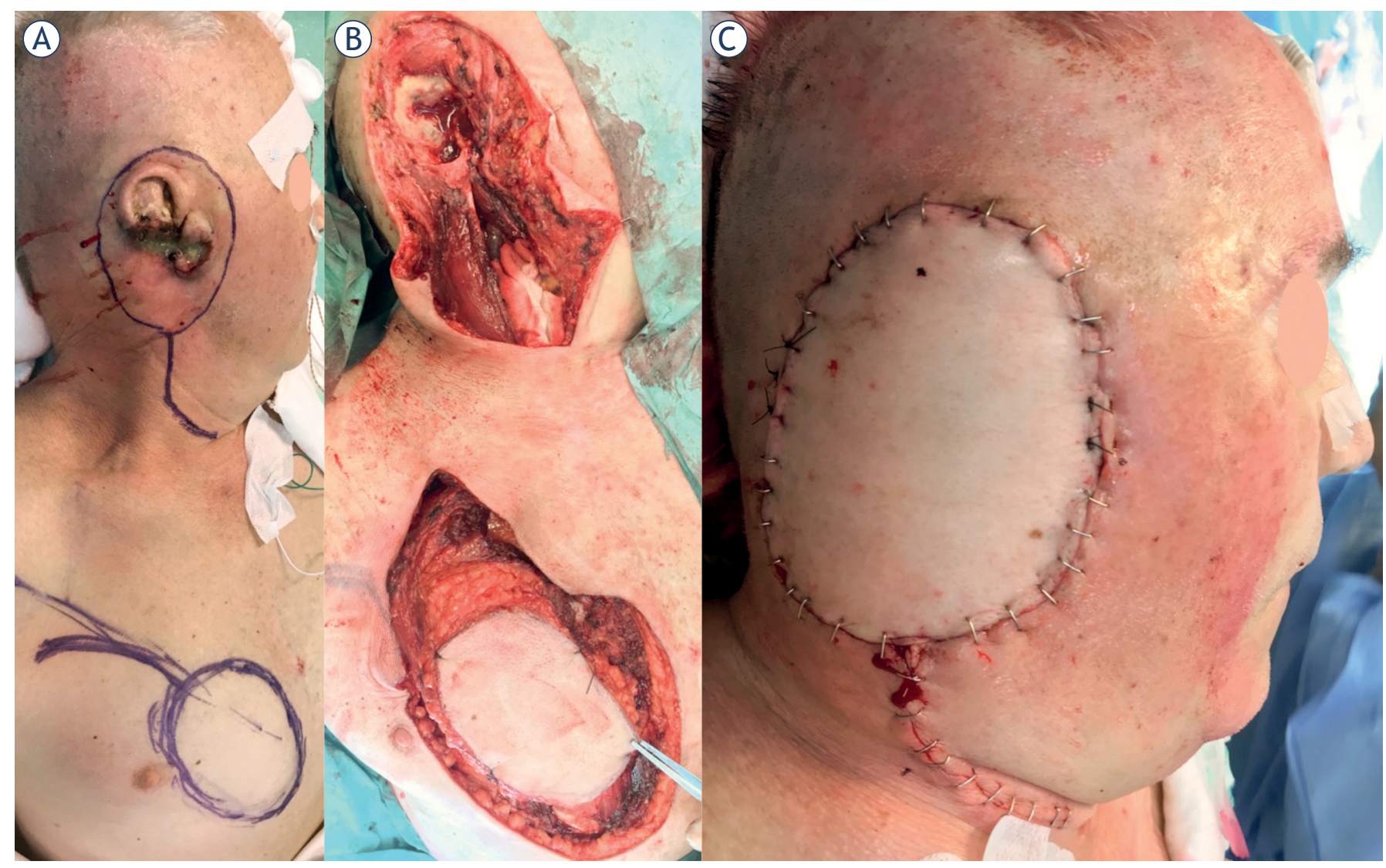

FIGURE 5. 76-year old male with locoregionally advanced external ear squamous cell carcinoma extending to the right lateral skull-base. The resection margin is outlined (A). Salvage surgery (i.e., after primary radical radiotherapy) involving lateral temporal bone resection with wide local excision, partial parotidectomy, ipsilateral selective neck dissection and pectoralis major myocutaneous flap reconstruction (due to recipient vessel insufficiency) were performed $(B, C)$.

\section{Discussion}

Our study presents 17 patients treated surgically with curative intent in 9 years at Slovenia's two main healthcare centres dealing with surgical and non-surgical head and neck cancer treatment. As many as 12 patients $(71 \%)$ had locoregionally advanced disease at the time of surgery.

The percentage $(10 \%)$ of patients included in the final analysis (17 patients) among initially collected data (177 patients) confirms that the lateral skullbase cancer is a rare entity, and data acquisition is arduous. The main reason is that this cancer can be classified under various ICD-10 diagnoses. Middle ear cancer is the only lateral skull-base cancer with a universal ICD-10 code (C30.1). Our study presents only the minority of lateral skull-base cancer; therefore, other ICD-10 diagnoses should be included. Despite the efforts, the list of included diagnoses in our study is possibly not exhaustive, and some cases with lateral skull-base involvement may have been diagnosed under other diagnoses (i.e., C44.3). As already proposed ${ }^{1,3}$, the establishment of the universal lateral skull-base cancer registry should be encouraged, which would overcome obstacles in prospective data analysis and alleviate multicentric research in this rare type of cancer.

Male predominance $(65 \%)$ and discharge as the most common initial symptom (71\%) in our group are consistent with the literature. Nevertheless, the average age at the cancer diagnosis $(M=74 S D=$ 13) was higher in our study. ${ }^{6}$

Set of histopathological types recorded in our patients are consistent with the literature which reports the predominance of squamous cell carcinoma. ${ }^{6}$ Nonetheless, its predominance over other cancer types is not as significant as reported in other studies. ${ }^{1}$ This is perchance due to the predominance $(57 \%)$ of basal cell carcinoma classified as aEEC, which present the majority ( $41 \%)$ of cases in our study. 

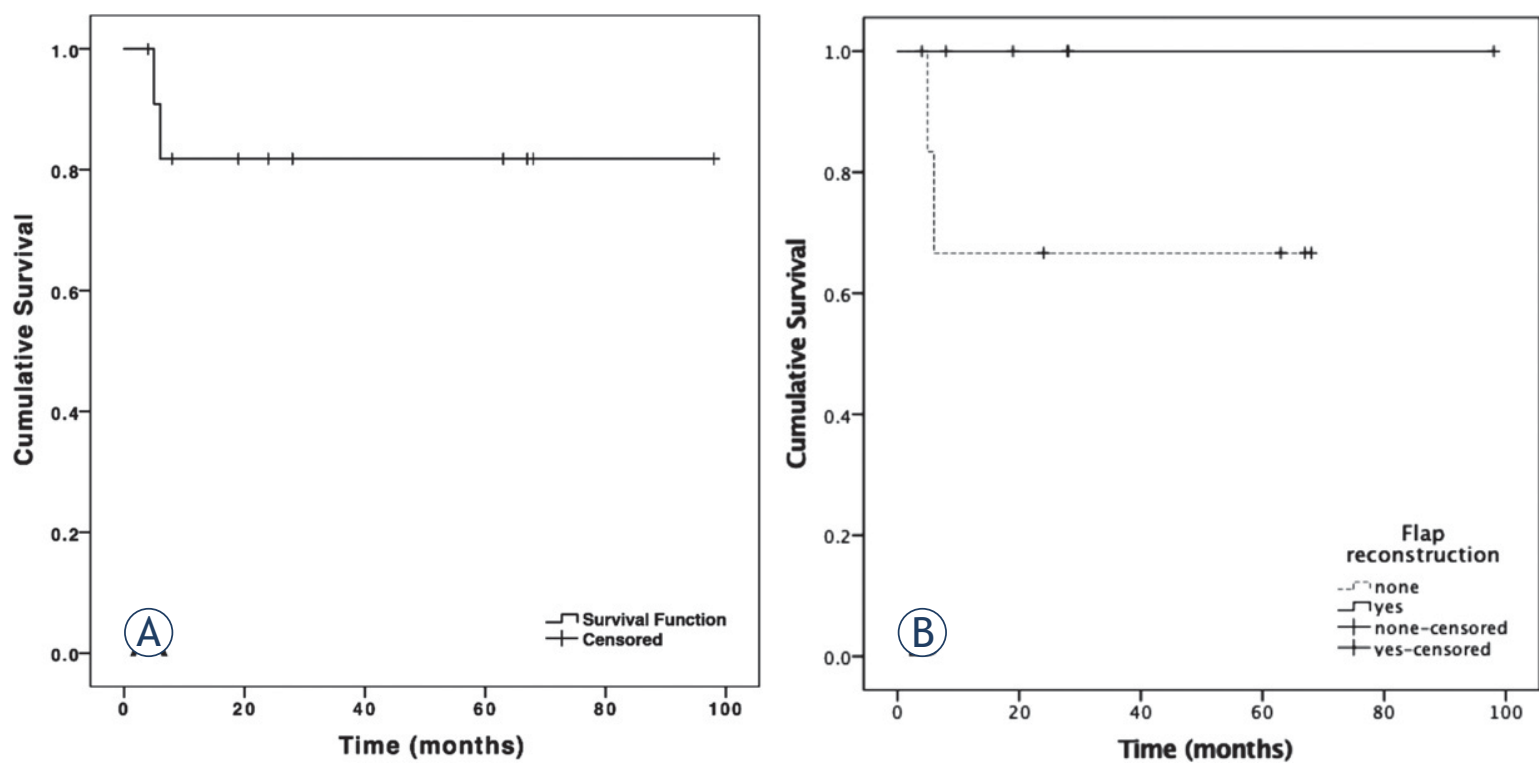

FIGURE 6. Kaplan-Meier analysis of overall survival of 12 patients with locoregionally advanced lateral skull-base cancer treated surgically with curative intent. (A) Kaplan-Meier analysis of 12 patients regardless of the reconstruction modality. Cumulative survival remained at $83 \%$ after six months. (B) Kaplan-Meyer analysis of 6 patients treated with flap reconstruction and six patients with other reconstruction modalities. Cumulative survival remained at $67 \%$ after six months.

The study focused on analysing surgically treated locoregionally advanced lateral skull-base cancer (i.e., TNM grades III and IV), which presented the vast majority of all cancers $(71 \%)$. These patients suffered from a severe mixed (i.e., sensorineural and conductive) hearing loss according to the hearing level measurements. Mostly, the extensive radical surgery was performed such as lateral temporal bone resection with obliteration $(66.7 \%)$, ipsilateral neck dissection (66.7\% selective, $8.7 \%$ modified radical) and parotidectomy (50\% partial, $8.3 \%$ total). In all patients with locoregionally advanced skin cancer of external ear (3 patients) and parotid cancers ( 2 patients), the extensive radical resection including skin prompted the tissue defect reconstruction with a major regional or free flap. Combination of extensive radical resection and flap reconstruction proved efficient since all of these patients are still alive. According to our experiences, ALT, RFFF, and PM can be harvested simultaneously while performing the lateral skullbase resection, which shortens surgery time but requires two surgical teams. None of our ALT, RFFF and PM failed, despite postoperative radiotherapy in 2 of these patients, consistent with the literature. ${ }^{15}$ Primary closure was the reconstruction of choice after resection of EACC or MEC since there was no large skin defect (i.e., blind sac external auditory canal closure and no pinna amputation).
Results show that our patients' local control and survival with locoregionally advanced cancer were high, especially in patients treated with flap reconstruction (Figure 6). The cancer-free survival rate of $100 \%$ was calculated on only four patients since others were not followed-up for at least five years. 83\% of alive patients at data collection cut-off confirms the favourable treatment outcome. This percentage is higher than the information collected in the pertinent literature $(58.7 \%)$, although our patients were considerably older than in other reports. ${ }^{1}$

Our study has certain limitations inherent to other retrospective studies. The calculation of the annual incidence of lateral skull-base cancer in our country could not be performed since the data involve only patients treated in a single tertiary otorhinolaryngology referral centre. Moreover, our study does not present patients treated with non-surgical modalities only. Namely, for a comprehensive overview of the field, it would be essential to consider other treatment modalities such as primary radio(chemo)therapy ${ }^{3}$ and also electrochemotherapy. ${ }^{16}$

\section{Conclusions}

Extensive radical resection of the tumour, adjacent tissues and structures of lateral skull-base should 
be planned in locoregionally advanced skin cancer of external ear and locoregionally advanced parotid cancer. The tissue defect should be reconstructed with tissue flap; therefore, otorhinolaryngologist treating this cancer should be experienced in free and regional flap elevation such as ALT, RFFF and PM. This surgical approach enables a high survival rate.

In locoregionally advanced primary external auditory canal cancer, the high survival rate is allowed with lateral temporal bone resection, obliteration and blind sac external auditory canal closure without amputation of the pinna, which offers the best chances for durable local control.

MEC is always locoregionally advanced if modified Pittsburgh staging system is applied, and the risk of tumour re-appearance is high despite extensive surgery and adjuvant treatment.

Collaboration within otorhinolaryngology subspecialists and oncologists is vital to treat lateral skull-base cancer. It is imperative to establish universal lateral skull-base cancer registry in tertiary healthcare centres involved in treating this disease.

\section{Acknowledgements}

To Tina Žagar and Aleš Matos for data collection and distribution. The study was financially supported by the Slovenian Research Agency (programme no. P3-0307).

\section{References}

1. Wierzbicka M, Niemczyk K, Bruzgielewicz A, Durko M, Klatka J, Kopeć T, et al. Multicenter experiences in temporal bone cancer surgery based on 89 cases. PLoS One 2017; 12: e0169399. doi: 10.1371/journal.pone.0169399

2. da Silva AP, Breda E, Monteiro E. Malignant tumors of the temporal bone - our experience. Braz J Otorhinolaryngol 2016; 82: 479-83. doi: 10.1016/j. bjorl.2015.09.010

3. Homer JJ, Lesser T, Moffat D, Slevin N, Price R, Blackburn T. Management of lateral skull base cancer: United Kingdom national multidisciplinary guidelines. J Laryngol Otol 2016; 130(Suppl 2): S119-24. doi: 10.1017/ S0022215116000542

4. Masterson L, Rouhani M, Donnelly NP, Tysome JR, Patel P, Jefferies SJ, et al. Squamous cell carcinoma of the temporal bone: clinical outcomes from radical surgery and postoperative radiotherapy. Otol Neurotol 2014; 35: 501-8. doi: $10.1097 / \mathrm{MAO} .0000000000000265$

5. Raghu M, Moumoulidis I, De R, Moffat D. Chondrosarcomas of the temporal bone: presentation and management. J Laryngol Otol 2004; 118: 551-5. doi: $10.1258 / 0022215041615272$

6. Sinha S, Dedmon MM, Naunheim MR, Fuller JC, Gray ST, Lin DT. Update on surgical outcomes of lateral temporal bone resection for ear and temporal bone malignancies. J Neurol Surg Part B Skull Base 2017; 78: 37-42. doi: $10.1055 / \mathrm{s}-0036-1584310$

7. Mehta GU, Muelleman TJ, Brackmann DE, Gidley PW. Temporal bone resec tion for lateral skull-base malignancies. J Neurooncol 2020; 150: 437-44. doi: $10.1007 / \mathrm{s} 11060-020-03445-4$
8. Moody SA, Hirsch BE, Myers EN. Squamous cell carcinoma of the external auditory canal: an evaluation of a staging system. Am J Otol 2000; 21: 582-8. doi: $10.1055 / \mathrm{s}-2007-994290$

9. Hirsch BE. Staging system revision. Arch Otolaryngol Neck Surg 2002; 128: 93-4. PMID: 11784271

10. Morita S, Mizumachi T, Nakamaru Y, Sakashita T, Kano S, Hoshino K, et al. Comparison of the University of Pittsburgh staging system and the eighth edition of the American Joint Committee on Cancer TNM classification for the prognostic evaluation of external auditory canal cancer. Int J Clin Oncol 2018; 23: 1029-37. doi: 10.1007/s10147-018-1314-3

11. Gupta R, Sandison A, Wenig BM, Thompson LDR. Data set for the reporting of ear and temporal bone tumors: explanations and recommendations of the guidelines from the International Collaboration on Cancer Reporting. Arch Pathol Lab Med 2019; 143: 593-602. doi: 10.5858/arpa.2018-0415-SA

12. Stell PM, McCormick MS. Carcinoma of the external auditory meatus and middle ear. Prognostic factors and a suggested staging system. J Laryngol Otol 1985; 99: 847-50. doi: 10.1017/s0022215100097796

13. Wei W, Qiu Y, Fang Q, Jia Y. Pectoralis major myocutaneous flap in salvage reconstruction following free flap failure in head and neck cancer surgery. $J$ Int Med Res 2019; 47: 76-83. doi: 10.1177/0300060518795530

14. Moncrieff MD, Hamilton SA, Lamberty GH, Malata CM, Hardy DG, Macfarlane $\mathrm{R}$, et al. Reconstructive options after temporal bone resection for squamous cell carcinoma. J Plast Reconstr Aesthetic Surg JPRAS 2007; 60: 607-14. doi: 10.1016/j.bjps.2006.11.005

15. Trojanowski P, Szymański M, Trojanowska A, Andrzejczak A, Szczepanek D, Klatka J. Anterolateral thigh free flap in reconstruction of lateral skull base defects after oncological resection. Eur Arch Otorhinolaryngol 2019; 276 3487-94. doi: 10.1007/s00405-019-05627-x

16. Bonadies A, Bertozzi E, Cristiani R, Govoni FA, Migliano E. Electrochemotherapy in skin malignancies of head and neck cancer patients: clinical efficacy and aesthetic benefits. Acta Derm Venereol 2019; 99: 1246-52. doi: 10.2340/00015555-3341 